\title{
Detection of SARS-CoV-2 with Solid-State CRISPR-Cas12a-Assisted Nanopores
}

\author{
Reza Nouri, Yuqian Jiang, Zifan Tang, Xiaojun Lance Lian, and Weihua Guan* \\ Cite This: https://doi.org/10.1021/acs.nanolett.1c02974 \\ Read Online
}

ABSTRACT: The outbreak of the SARS-CoV-2 caused the disease COVID-19 to spread globally. Specific and sensitive detection of SARS-CoV-2 facilitates early intervention and prevents the disease from spreading. Here, we present a solid-state CRISPR-Cas12aassisted nanopore (SCAN) sensing strategy for the specific detection of SARS-CoV-2. We introduced a nanopore-sized counting method to measure the cleavage ratio of reporters, which is used as a criterion for positive/negative classification. A kinetic cleavage model was developed and validated to predict the reporter size distributions. The model revealed the trade-offs between sensitivity, turnaround time, and false-positive rate of the SARS-CoV-2 SCAN. With preamplification and a $30 \mathrm{~min}$ CRISPR Cas12a assay, we achieved excellent specificity against other common human
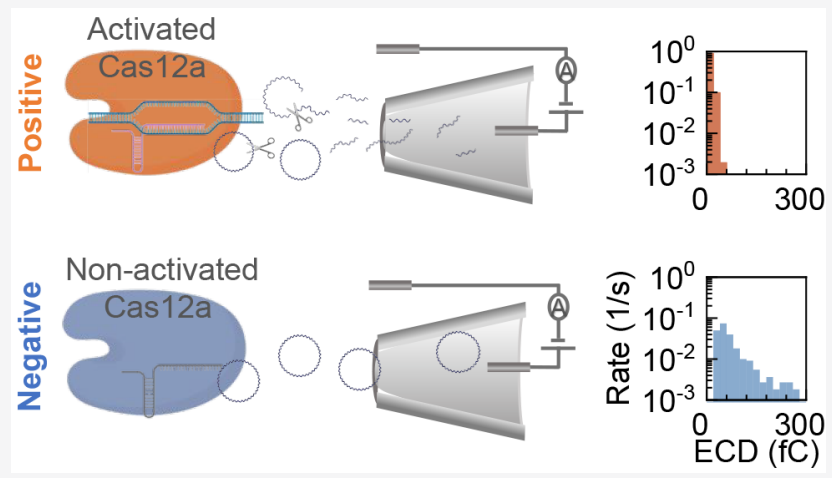
coronaviruses and a limit of detection of 13.5 copies $/ \mu \mathrm{L}(22.5 \mathrm{aM})$ of viral RNA at a confidence level of $95 \%$. These results suggested that the SCAN could provide a rapid, sensitive, and specific analysis of SARS-CoV-2.

KEYWORDS: CRISPR, Cas12a, SARS-CoV-2, COVID-19, nanopore

$\mathrm{C}$ oronavirus disease 2019 (COVID-19) caused by severe acute respiratory syndrome coronavirus 2 (SARS-CoV-2) is an ongoing pandemic throughout the world. ${ }^{1,2}$ To facilitate the management and containment of the disease, reliable, rapid, and accessible testing is required. While numerous diagnostic strategies such as sequencing ${ }^{3-6}$ and antibody testing $^{7-9}$ have been introduced for SARS-CoV-2 detection, nucleic acid testing (NAT) methods, primarily quantitative real-time PCR with reverse transcription (RT-qPCR), are the current gold standard. ${ }^{10,11}$ The recent development of the clustered regularly interspaced short palindromic repeats (CRISPR)-based methods started a new path toward molecular diagnosis. ${ }^{12}$ Particularly, the discovery of the collateral cleavage of Cas proteins such as Cas12 and Cas13 made it possible to translate the sequence-specific targeting of detectable signals. These discoveries have led to a variety of CRISPR-mediated biosensors. ${ }^{13-21}$ These CRISPR-based methods often incorporate an amplification process such as polymerase chain reaction (PCR), ${ }^{22,23}$ loop-mediated isothermal amplification (LAMP), ${ }^{24}$ or recombinase polymerase amplification (RPA) $)^{25,26}$ to enhance the starting molecule population. ${ }^{14,27}$ Amplification-coupled CRISPR-Cas detection has been shown to be highly sensitive (as low as the aM

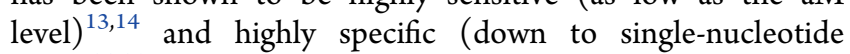
level). ${ }^{28,29}$ Due to their outstanding sensing performance, CRISPR-based systems have been adopted for SARS-CoV-2 detection amid the ongoing COVID-19 pandemic. ${ }^{10,23,30-38}$
So far, most of the CRISPR-based methods use fluorescent, bioluminescent, or colorimetric reporters for readouts, which are easy to operate, sensitive, and convenient. ${ }^{39}$ As alternatives to the optical readout, electronic-based methods such as electrochemical $^{17,40-42}$ and field-effect ${ }^{43}$ have also been investigated due to their integration and miniaturization potential. One of the intriguing electronic readout systems utilized for CRISPR-based detection is the nanopore sensor. $^{44,45}$ The single-molecule sensitivity of the nanopore sensors has made them a promising candidate for CRISPRbased detection. We previously demonstrated a solid-state CRISPR-Cas12a-assisted nanopore (SCAN) sensor for sequence-specific recognition of HIV-1. ${ }^{46}$ While we demonstrated that the SCAN can detect target DNA concentrations above $10 \mathrm{nM}$ within $1 \mathrm{~h}$, detecting concentrations lower than $10 \mathrm{nM}$ with a fast turnaround time would likely require preamplification steps.

In this work, we developed a reverse-transcription amplification-coupled SCAN device for rapid, highly sensitive, and highly specific detection of SARS-CoV-2 viral RNAs. The

Received: August 3, 2021

Revised: September 16, 2021 
a)
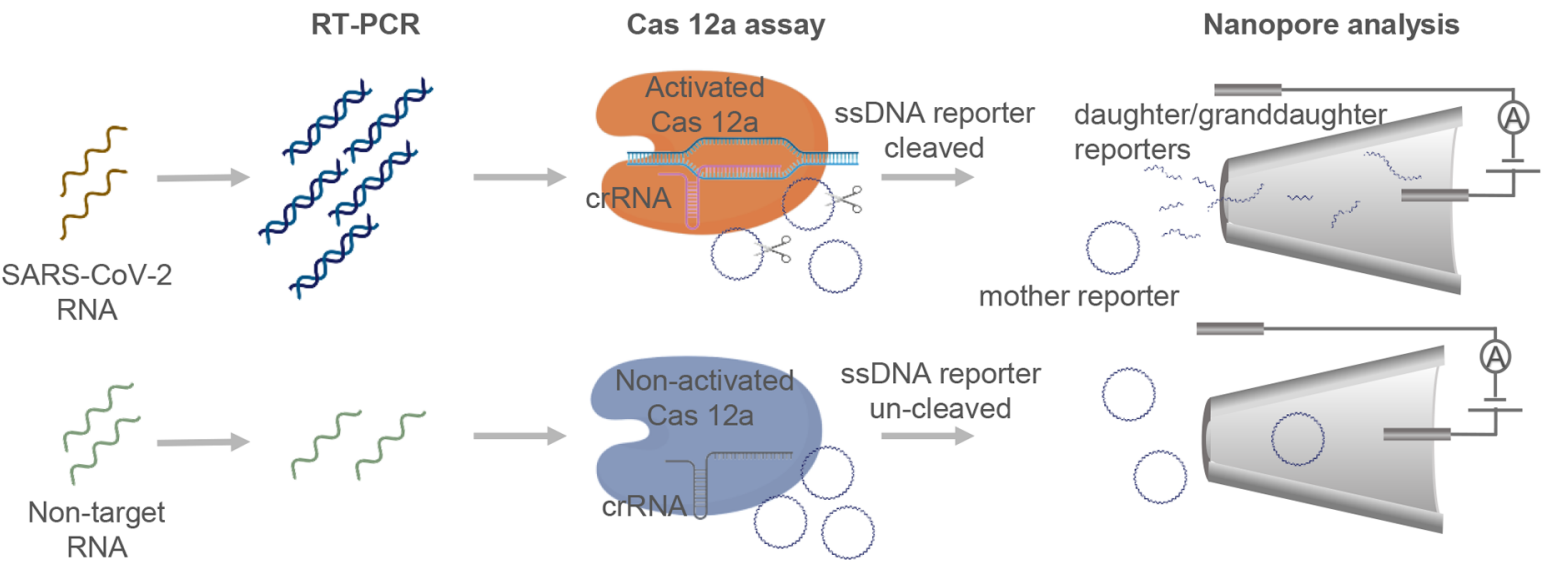

b)

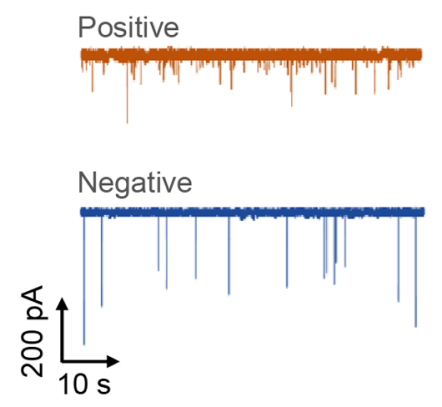

c)

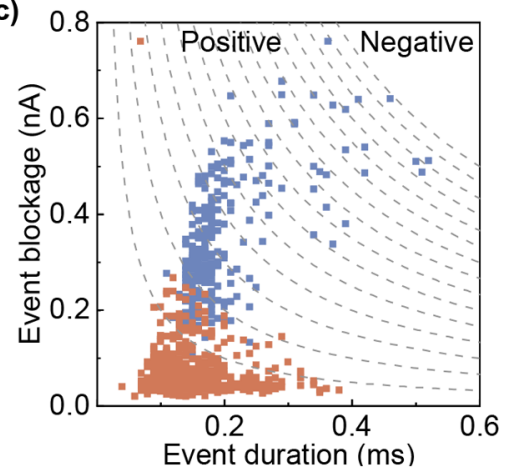

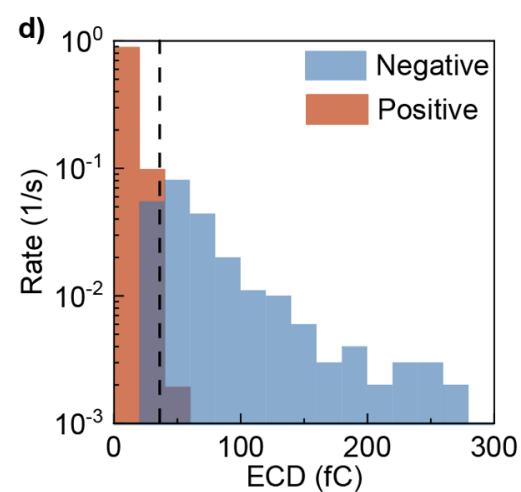

Figure 1. (a) Schematic of solid-state CRISPR-Cas12a-assisted nanopore (SCAN) sensor. The process starts with a preamplification step, followed by CRISPR assay and nanopore analysis. In a positive case (upper side), the trans-cleavage activity of the Cas12a after activation cause degradation of the circular ssDNA reporters, resulting in reduced reporter size. In a negative case, the Cas12a is not activated in the absence of target dsDNA, and thus the ssDNA reporters are not cleaved. (b) Examples of a typical ionic current trace for a positive and negative case. (c) Duration and blockage of translocation events for a positive and negative case. The lines represent equivalent ECD lines from 20 to $300 \mathrm{fC}$ (with a bin size of 20 fC). (d) Event rate distribution at different ECD values. The right side of the dashed line represents the uncleaved region.

method used an improved nanopore sized counting approach to examine the reporter size distributions and their relative abundance. We showed that the cleavage ratio of the intact circular ssDNA reporters could be quantified by the SCAN, which is used as a criterion for classifying the test as positive or negative. To guide the experiments, we developed a kinetic model to compute the reporter length distribution as a function of the cleavage reaction time. This experimentally validated model revealed the trade-offs between sensitivity, turnaround time, and false-positive rate of the SARS-CoV-2 SCAN. With a preamplification step and $30 \mathrm{~min}$ CRISPR Cas12a assay, we achieved a limit of detection (LoD) of 13.5 copies $/ \mu \mathrm{L}(22.5 \mathrm{aM})$ of SARS-CoV-2 viral RNA at a confidence level of $95 \%$. The SARS-CoV-2 SCAN has also shown excellent specificity against three other common human coronaviruses. Our results suggested that the SCAN could provide a rapid, sensitive, and specific analysis of SARS-CoV-2.

\section{RESULTS AND DISCUSSION}

Working Principle of Nanopore-Sized Counting. Figure 1a illustrates the working scheme of the SARS-CoV-2 SCAN using nanopore-sized counting. There were three streamlined steps: reverse transcription and amplification, Cas12 assay, and nanopore-based molecule classification and counting. In the first step, a one-step reverse-transcription polymerase chain reaction (RT-PCR) of SARS-CoV-2 RNA was conducted to improve the overall sensitivity of the system. ${ }^{31,32,34}$ After amplification, the complementary DNA (cDNA) amplicons were introduced to the sequence-specific CRISPR RNA (crRNA) and Cas12a ribonucleoprotein mixture (a.k.a., RNP). Upon specific cDNA binding, Cas12a could conduct collateral cleavage on the surrounding ssDNA reporters. ${ }^{39}$ We used circular M13mp18 single-stranded DNA (ssDNA) as the reporter in this study which is widely available and has an excellent signal-to-noise ratio in nanopore measurement. In the trans-cleavage process, the mother circular ssDNA reporters could be digested into daughter linear ssDNAs, and the daughter reporters could be further digested into granddaughter reporters (positive case in Figure 1a). However, if SARS-CoV-2 viral RNAs were not present in the analyte solutions, then the Cas12a remains inactive and will not degrade the mother circular ssDNA reporter (negative case in Figure 1a).

These uncleaved mother reporters and multigenerational cleaved daughter reporters were then counted and classified by a glass nanopore sensor to infer their size and concentration distributions. Figure $1 \mathrm{~b}$ shows two representative ionic current time traces for a positive sample and a negative sample (no target control), using a glass nanopore with a diameter size less than $10 \mathrm{~nm}$ (Figure S1a,b). For the positive sample (presence of SARS-CoV-2 RNA), it is apparent that molecular translocation events become more frequent but have less current 
a)

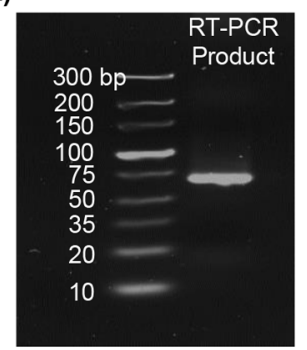

b)

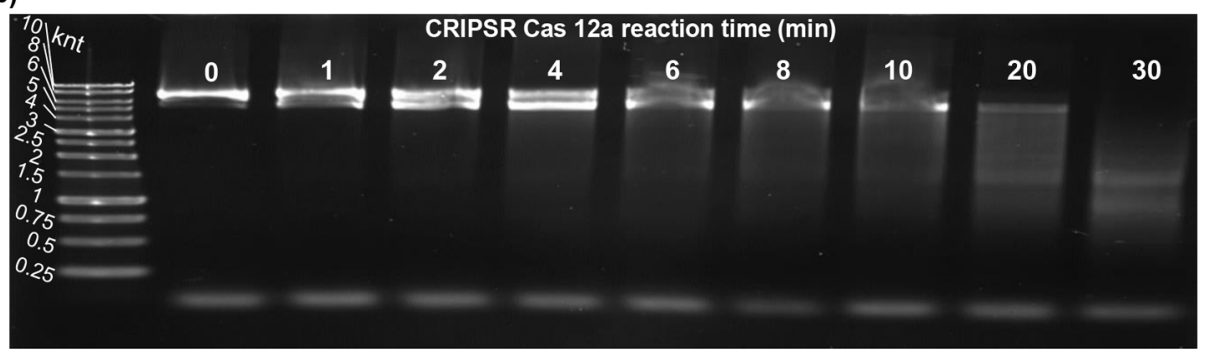

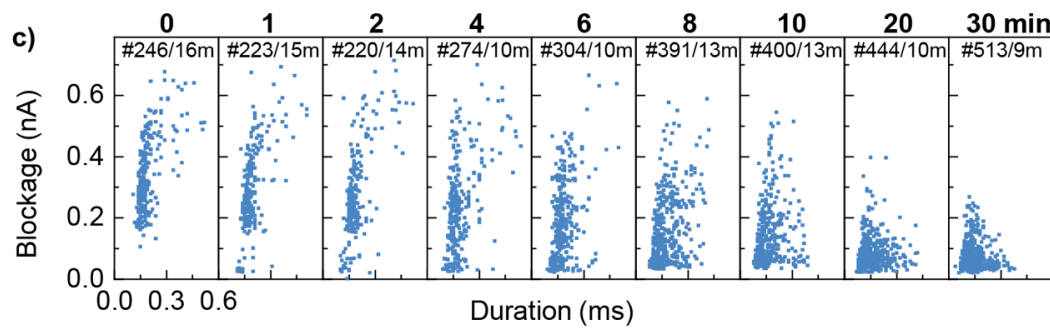

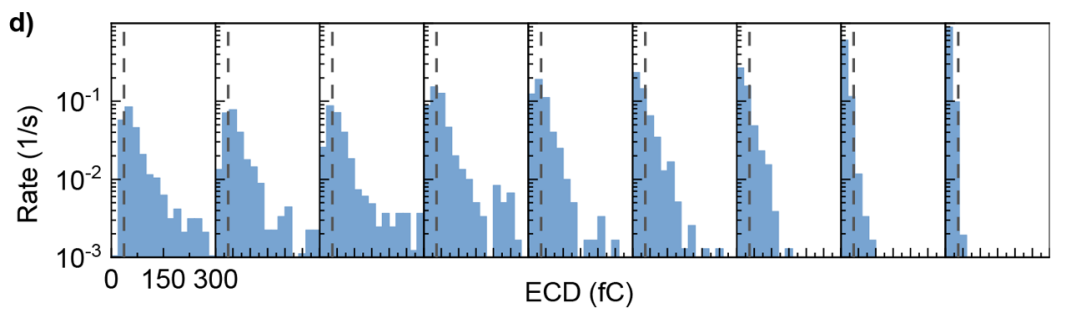

e)

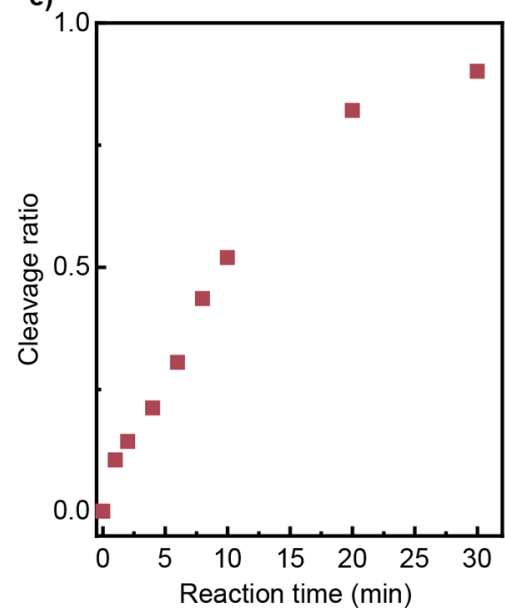

Figure 2. (a) Gel electrophoresis results of the RT-PCR products validating the length of amplicons as $67 \mathrm{bp}$. (b) Gel electrophoresis results of Cas12a assay products at different reaction times from 0 to $30 \mathrm{~min}$. In all cases, the nonactivated Cas12a and reporter concentrations were fixed at 30 and $2.1 \mathrm{nM}$, respectively. (c) Current drop and dwell times of the ssDNA reporter translocation events at different reaction times through the glass nanopore under $400 \mathrm{mV}$ bias. The buffer salt concentration was fixed at $1 \mathrm{M}$. The total number of events and nanopore reading time is shown for each case. (d) Event rate distribution at different ECD values. The area right to the dashed line represents the uncleaved region. (e) Calculated values for reporter cleavage ratios at different reaction times.

blockage magnitude as compared to the negative sample. This is because the mother circular reporters were cleaved to many smaller daughter linear reporters. Our previous work analyzed the resulting reporter concentration by nanopore digital counting without taking the daughter reporter size distribution into consideration. ${ }^{30}$ This assumption is not exactly accurate if the reaction time is short. To further analyze the daughter reporter size distribution and its relative abundance, we here adopted a nanopore-sized counting method. First, the conventional event duration versus blockage was obtained from the ionic current time trace data (Figure S1b). As shown in Figure 1c, it is evident that the event duration and blockage in a positive case are smaller than in a negative case. Second, we classified each event based on its event charge deficit (ECD), which is used as the molecule size approximation. ${ }^{40}$ The ECD is defined as ECD $=\int_{\text {event }} \Delta I(t) \mathrm{d} t \cong \Delta I \tau,{ }^{41}$ where $\Delta I$ and $\tau$ are the duration and blockage of each event, respectively. It was previously demonstrated that ECDs of DNA translocations with the same length are identical regardless of whether the molecules are in a linear, circular relaxed, or supercoiled form. ${ }^{40}$ An ECD bin size of $20 \mathrm{fC}$ was used in this study unless otherwise stated. Third, the event rate of each ECD subpopulation was obtained by normalizing subpopulation event numbers by the nanopore reading time (Figure 1d). This enables us to quantify the reporter subpopulation concentration through $R_{i}=C_{i} \alpha N_{\mathrm{A}}$, where $N_{\mathrm{A}}$ is the Avogadro constant and $\alpha$ is usually referred to as the capture rate. $^{42-45}$
The mean $(\mu)$ and standard deviation $(\sigma)$ of the ECD value in the negative cases was used to establish an ECD threshold (ECDt $=\mu-2 \sigma$, dashed line in Figure 1d). An event must have an ECD larger than ECDt to be classified as an intact mother reporter (i.e., to the right of the dashed line in Figure 1d). To quantify the percentage of the mother reporters being cleaved into daughter reporters, we defined the cleavage ratio (CR) as the ratio between the cleaved mother reporter $\left(C_{0}-\right.$ $\left.C_{\text {uncleaved }}\right)$ to the total initial mother reporter $\left(C_{0}\right)$. This cleavage ratio can be experimentally obtained by evaluating the aggregated event rate as

$$
\mathrm{CR}=1-\sum_{i} R_{p i} / \sum_{j} R_{n j}
$$

in which the event rate summation is over all events with ECD larger than ECDt (thus representing the intact mother reporter concentration in the system) and $n$ and $p$ denote the negative and positive cases, respectively. The increase in the cleavage ratio of a testing sample would confirm the existence of the target viral RNA.

SARS-CoV-2 Cas12a Assay Validation. Prior to the Cas12a assay, one-step RT-PCR was conducted to increase the number of molecules and boost the signal. We utilized the primers designed by the United States Centers for Disease Control and Prevention (CDC) targeting the N2 region of the SARS-CoV-2. ${ }^{46}$ We conducted a real-time RT-PCR with $2 \times$ $10^{5}$ copies/ $\mu \mathrm{L}$ SARS-CoV-2 viral RNAs for a duration of 45 cycles. The fluorescent signal confirmed the amplification after 
20 cycles (Figure S2). To further confirm the amplicon product, we conducted gel electrophoresis of the RT-PCR products, which showed a sharp band at $67 \mathrm{bp}$, as expected with our RT-PCT primer design (Figure 2a).

Afterward, we conducted the SARS-CoV-2 specific Cas12a assay with a reaction time ranging from 0 to $30 \mathrm{~min}$. The reaction was stopped by adding the DNA gel loading dye $(6 \times)$, which contained ethylenediaminetetraacetic acid (EDTA). Figure $2 \mathrm{~b}$ presents the gel electrophoresis results of the Cas12a assay. We observed several important features. First, the mother reporter appeared in a double band around $7 \mathrm{kbp}$. This is due to the fact that electrophoretic mobility of DNA in gels could also be affected by the conformation of the DNA. ${ }^{47}$ Second, the daughter reporters become visible after $2 \mathrm{~min}$, indicating the cleavage of mother reporters. Third, the primers were observed as a blurred short band in all cases. Fourth, as we increased the reaction time, more mother reporters were cleaved. At $30 \mathrm{~min}$, the 7.2 kilonucleotides (knt) band of the uncleaved mother ssDNA become barely visible.

To examine the Cas12a cleavage kinetics at a much longer time scale, we conducted another test by intentionally extending the Cas12a reaction time up to $24 \mathrm{~h}$. We found that all mother and prior generation daughter reporters were completely cleaved to be less than $250 \mathrm{nt}$ after $24 \mathrm{~h}$ (Figure S3). This suggests that the trans-cleavage activities indiscriminately and continuously affect both the mother reporters and the partially cleaved daughter reporters. As a control, we also conducted the Cas12a assay for no target samples to confirm that no degradation of the mother reporters would occur in the absence of SARS-CoV-2 amplicons (Figure S4).

Highly Sensitive Nanopore Measurement of the Cleavage Ratio. After validating the Cas12a assay with gel electrophoresis, we set out to conduct SARS-CoV-2 detection with the glass nanopore. We conducted the Cas12a assay with different reaction times from 0 to $30 \mathrm{~min}$ and recorded the reporter translocations through the nanopore under $400 \mathrm{mV}$ bias (Figure S5). In our glass nanopore measurement, DNAs with sizes less than $100 \mathrm{bp}$ are often too small to be detected. Those significantly cleaved reporters with lengths less than 100 bp and RT-PCR amplicons (67 bp) could not contribute to the detected signals. In addition, in our previous study, ${ }^{48}$ we showed that the other components in the assay, such as Cas12a proteins, do not create signals in the nanopore experiment. Therefore, all the signals in the nanopore measurements are caused by the reporters with a length above the detectable threshold (a few hundred nucleotides).

Figure $2 \mathrm{c}$ shows the extracted translocation dwell time versus ionic current blockage at each reaction time. A clear shift of the blockage-duration distribution was observed when increasing the reaction time, indicating the changing populations of differently sized reporters. To quantify the abundance of differently sized reporters, we used an ECD bin size of $20 \mathrm{fC}$ to classify the events into different subpopulations and calculated its corresponding event rate. Figure $2 \mathrm{~d}$ shows the event rate distribution for all subpopulations. As shown, the event rate of larger ECDs (longer reporters) is reducing as the cleavage reaction goes, whereas the event rate of smaller ECDs (shorter reporters) is increasing. Since the concentration of the analyte could be quantified by the event rate in the nanopore experiment, these measurements give us the capability to quantify the relative abundance of differently sized reporters.
To quantify the cleavage ratio at different reaction times, we utilized the nanopore-sized counting method. The case at 0 min of reaction was considered as the negative case to establish the ECDt. The cleavage ratio (CR) at each reaction time was then obtained by using eq 1 . As shown in Figure 2e, the CR was at 0.12 after 1 min of reaction and increased with increasing reaction time. For instance, $\mathrm{CR}$ was measured as 0.92 at $30 \mathrm{~min}$ of reaction. As the reaction time increases, more daughter and granddaughter reporters would be created, which increases the possibility of multiturn cleavage. Therefore, the cleavage ratio increases at a lower rate as we increase the reaction time. It is notable that we were able to detect the cleavage activity after $1 \mathrm{~min}$ in nanopore reading, whereas no cleavage was barely visible in the gel after $1 \mathrm{~min}$ of reaction (Figure 2b). This indicates that the nanopore is a much more sensitive readout system for CR measurement.

Cleavage Numerical Modeling and Validation. In order to guide our experiments for rapid and sensitive detection of the cleavage ratio using nanopore experiment, we sought to develop a model to estimate the distribution of reporter length over the trans-cleavage process. At the start of the reaction, we assumed that each mother reporter has an identical length of $L_{\mathrm{m}}$. As the reaction starts, the reporters (including mother and daughter reporters) were randomly picked by the activated Cas12a. The probability that a reporter was picked and cleaved by the Cas12a was proportional to its cross-sectional area $R_{\mathrm{g}}^{2}$, where $R_{\mathrm{g}}$ is the gyration radius of the DNA coil. It has been shown that the gyration radius of DNA is proportional to the square root of its length $\left(L^{1 / 2}\right){ }^{42}$ Therefore, the longer the reporter was, the more chance that it was bounded to and cleaved by the Cas12a. The reporter was cut into two parts randomly. We assumed a normal distribution to model the cleavage position in a report. The velocity of the cleavage was modeled by $v(t)=k_{\text {cat }} \frac{\left[E_{0}\right][S(t)]}{K_{\mathrm{M}}+[S(t)]}$, in which $\left[E_{0}\right]$ is the initial concentration of enzyme (activated Cas 12a), $[S(t)]$ is the substrate concentration (mother and daughter reporters), and $k_{\text {cat }}$ and $K_{\mathrm{M}}$ are the catalytic rate and the Michaelis constant, respectively. We used previously reported $k_{\text {cat }}$ and $K_{\mathrm{M}}$ values of $0.6(1 / \mathrm{s})$ and $2.7 \times 10^{-6} \mathrm{M}$, respectively. ${ }^{49}$ This model was numerically implemented in a customized MATLAB code (see Figure S6 for model flowchart and results).

In order to validate this numerical model for predicting the reporter size distribution after reaction, we conducted the Cas12a assay at three enzyme concentrations (7.5, 15, and 30 $\mathrm{nM}$ ) with reaction times ranging from $0 \mathrm{~min}$ to $24 \mathrm{~h}$. Figure $3 \mathrm{a}$ presents the gel electrophoresis results. As expected, a higher enzyme concentration indeed results in faster cleavage activity since the cleavage velocity is proportional to the enzyme concentration. We extracted the length distribution of the reporter from the gel images by measuring the normalized grayscale values using ImageJ software. ${ }^{50,51}$ The normalized reporter length distributions were then overlaid with the results produced by our model. As shown in Figure 3b, shorter daughter reporters were produced at a fixed reaction time as enzyme concentration was increased. Also, both model and gel results showed that the mother reporters were cleaved entirely after $24 \mathrm{~h}$. The distribution of reporter length captured by our model is consistent with the gel electrophoresis results, which validates our model.

Trade-Off among Sensitivity, Reaction Time, and False-Positive Rate. Since the cleavage ratio (CR) was used 
a)
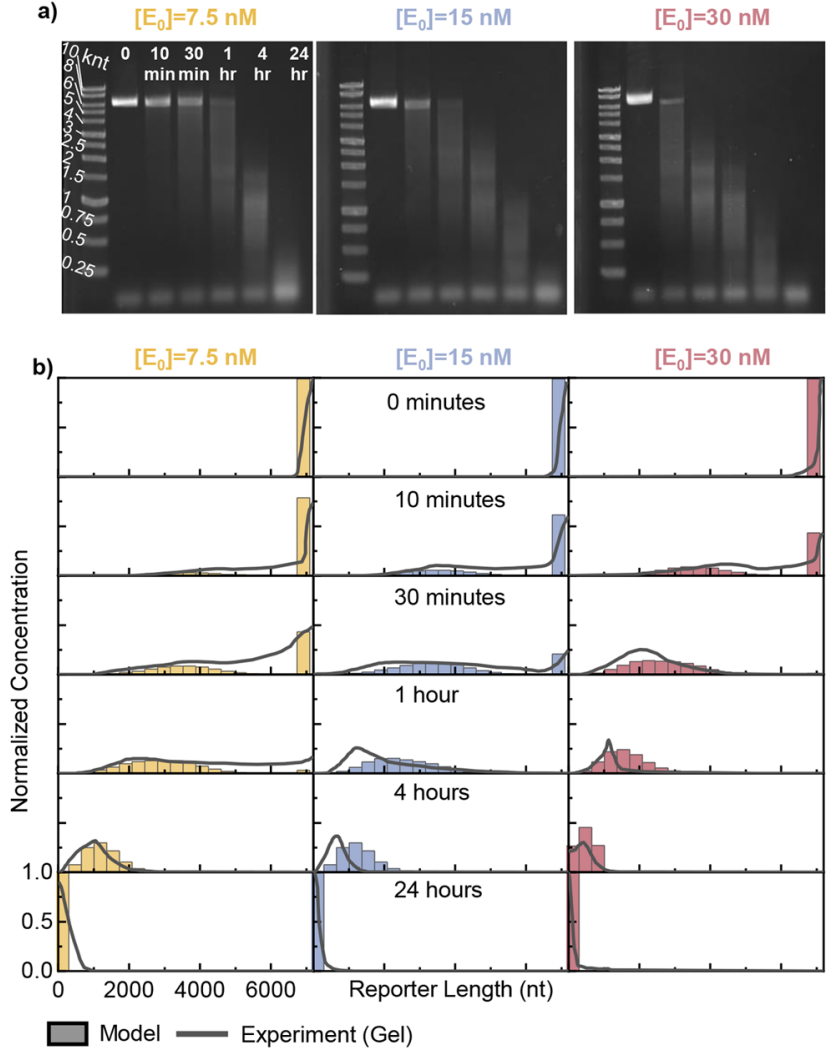

Figure 3. (a) Gel electrophoresis results of the Cas12a assay at three different initial enzyme concentrations (7.5, 15, and $30 \mathrm{nM}$ ) and different reaction times (from $0 \mathrm{~min}$ to $24 \mathrm{~h}$ ). (b) Comparison between the reporter length distribution captured by the model and gel electrophoresis. The normalized grayscale value of the gel results was measured by Image J software for the estimation of the reporter concentration.

to distinguish a positive and a negative sample, we calculated the CR with the model-produced length distributions. The model-derived $\mathrm{CR}$ values were then compared with the nanopore-measured $\mathrm{CR}$ values using the sized counting method (eq 1). We measured the $\mathrm{CR}$ at different enzyme concentrations $(7.5,15$, and $30 \mathrm{nM})$ with reaction times ranging from 0 to $30 \mathrm{~min}$. As shown in Figure $4 \mathrm{a}$, the model predicted CR values agree excellently with that measured by the nanopore.

With the capability to calculate the $\mathrm{CR}$ at varying activated Cas $12 \mathrm{a}$ and reaction time, we were able to estimate the sensitivity and turnaround time of the SCAN system at any given $\mathrm{CR}$ threshold $\left(\mathrm{CR}_{t}\right)$ for a positive call. Figure $4 \mathrm{~b}$ presents the minimal required reaction time versus the activated Cas12a enzyme concentration. The activated Cas12a enzyme concentration is equal to the smaller values between SARS-Cov-2 amplicons and nonactivated Cas $12 \mathrm{a}$ concentration in the system. As shown in Figure $4 b$, at any given $\mathrm{CR}_{t}$ for a positive call, there is a trade-off between turnaround time and sensitivity. More reaction time was required when decreasing the activated Cas12a enzyme concentration. For instance, at $\mathrm{CR}_{t}=0.1$, more than $45 \mathrm{~min}$ will be required to detect $1 \mathrm{nM}$ amplicons, whereas $0.45 \mathrm{~min}$ is sufficient with $100 \mathrm{nM}$ amplicons. In contrast, while increasing the $\mathrm{CR}_{t}$ could help to reduce the false-positive rate, it would increase the required minimal reaction time at any given amplicon concentrations. In fact, the CR is proportional to the product of cleavage velocity

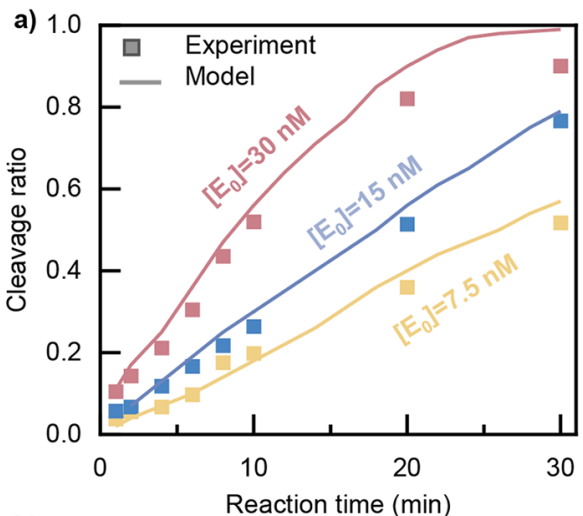

b)

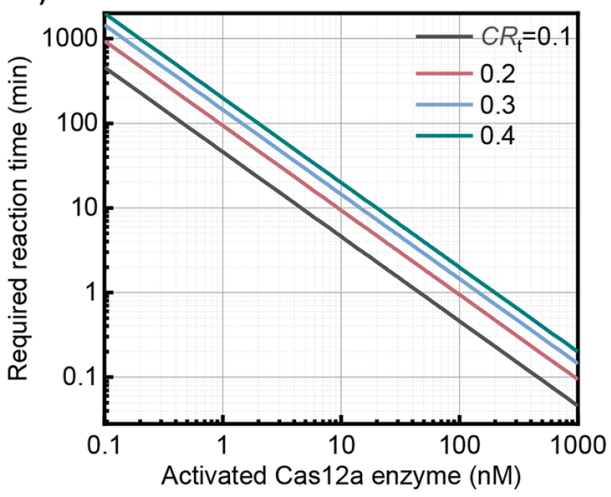

Figure 4. (a) Measured cleavage ratio of the ssDNA reporter by the sized counting method (experiment) and the numerical model at different reaction times. The outcome of the model and nanopore experiment is in good agreement at three different enzyme concentrations $(7.5,15$, and $30 \mathrm{nM})$. (b) Required reaction time versus the activated Cas12a enzyme concentration (from 0.1 to 1000 $\mathrm{nM}$ ) at four different CR thresholds values.

and reaction time $\left(T_{\mathrm{r}}\right), \mathrm{CR}=v T_{r}$. The cleavage velocity $v$ is proportional to the activated Cas 12 a concentration $C_{\text {enzyme }}$ as $v$ $=\beta \mathrm{C}_{\text {enzyme }}$. Therefore, one can see that $C_{\text {enzyme }} T_{\mathrm{r}} / \mathrm{CR}$ should equal to the constant coefficient $\beta$. This relationship suggests there is a trade-off between sensitivity $\left(C_{\text {enzyme }}\right)$, reaction time $\left(T_{\mathrm{r}}\right)$, and false-positive rate $(\mathrm{CR})$. With a fixed $C_{\text {enzyme }}$, a higher CR (less false-positive) calls for a longer reaction time $T_{\mathrm{r}}$. With a fixed $\mathrm{CR}$, reducing the $C_{\text {enzyme }}$ (better sensitivity) also requires a longer reaction time $T_{\mathrm{r}}$ (longer turnaround). By coupling with a preamplification step, the $C_{\text {enzyme }}$ can be effectively enhanced and thus significantly reduce the required $T_{\mathrm{r}}$ (turnaround time).

Analytical Specificity and Sensitivity of SARS-CoV-2 SCAN. We then went on to evaluate the analytical sensitivity and specificity of SARS-CoV-2 SCAN. We used heatinactivated SARS-CoV-2 RNA samples at different concentrations ranging from 2 to 200 copies $/ \mu \mathrm{L}$. In addition, three other human coronaviruses (229E, NL63, and OC43) with a concentration of $5 \times 10^{5}$ copies $/ \mu \mathrm{L}$ were used as the nontarget negative controls to evaluate the specificity. Each sample ( 5 $\mu \mathrm{L}$ ) was first amplified by RT-PCR for 45 cycles (Figure S7). The product of the RT-PCR was added to $30 \mathrm{nM}$ nonactivated Cas12a. The Cas12a cleavage assay was conducted at $37^{\circ} \mathrm{C}$ for $30 \mathrm{~min}$. Afterward, the nanopore-sized counting was conducted to determine the cleavage ratio. A positive/negative call was subsequently derived by comparing the obtained cleavage ratio with $\mathrm{CR}_{t}$ (dashed line in Figure 5a). The $\mathrm{CR}_{t}$ was defined in our experiment as $\mu_{0}+2 \sigma_{0}$ (0.089), where $\mu_{0}$ 

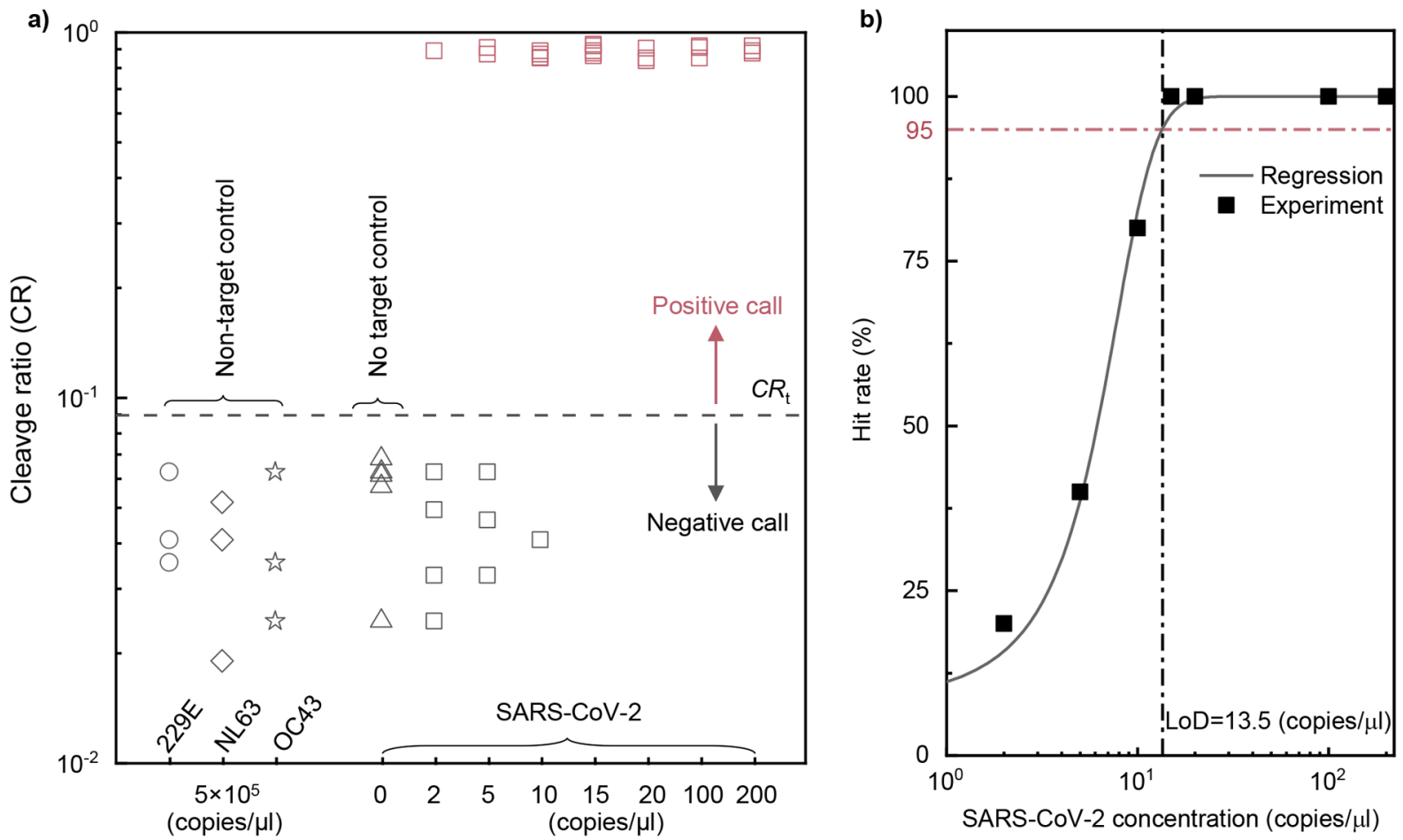

Figure 5. (a) Cleavage ratio of three different human coronavirus samples (for specificity test) and SARS-CoV-2 (for sensitivity test) with different input RNA concentrations. Five repeats were conducted for lower concentrations (less than 20 copies/ $\mu \mathrm{L}$ ), and three were tested for higher concentrations (more than 20 copies $/ \mu \mathrm{L}$ ) and nontarget control samples. The cleavage ratios were measured using the nanopore sized counting experiment. The average and standard deviation of the CR values of 5 negative target controls were measured to identify the threshold for separating positive from negative calls. Any cases with a CR below $\mathrm{CR}_{t}=\mu+2 \sigma(0.089)$ were classified as negatives. (b) Hit rate percentage at different concentrations of SARS-CoV-2 RNA. The LoD was estimated as 13.5 copies $/ \mu \mathrm{L}$ at a $95 \%$ confidence level.

(0.054) and $\sigma_{0}(0.017)$ are the mean and standard deviation of the cleavage ratio obtained from no target controls (i.e., SARSCoV-2 sample at zero concentrations). Figure 5a presents the cleavage ratios of all SARS-CoV-2 and nontarget human coronavirus samples. As shown, the cleavage ratios for all nontarget human coronavirus samples (circle, diamond, and star symbols in Figure 5a) are less than $\mathrm{CR}_{t}$ and were correctly classified as negatives. This confirmed that the SCAN sensor has an excellent specificity against SARS-CoV-2.

For the serially diluted SARS-CoV-2 samples (square symbols in Figure 5a), we found that concentrations higher than 15 copies $/ \mu \mathrm{L}$ were classified correctly as positives since their cleavage ratios are much larger than $\mathrm{CR}_{t}$. The high cleavage ratio in these samples indicates the majority of the mother reporters were cleaved after $30 \mathrm{~min}$ of reaction. However, we started to observe false negatives results in samples with concentrations lower than 15 copies $/ \mu \mathrm{L}$. For instance, 1 out of 5 samples at a concentration of 10 copies $/ \mu \mathrm{L}$ and 4 out of 5 samples at 2 copies/ $\mu \mathrm{L}$ were misclassified as negatives. To estimate the LoD of SARS-CoV-2 SCAN, we examined the hit rate at each different SARS-CoV-2 concentration. The hit rate is defined as the number of replicates with a detected outcome per the total number of replicates tested. ${ }^{52}$ As shown in Figure 5 b, the hit rate started to roll off from 1 to 0.8 when the concentration decreased from 15 to 10 copies $/ \mu \mathrm{L}$. We fit the experimental hit rate data with a logistic curve (Figure $5 \mathrm{~b}){ }^{53,54}$ On the basis of the fitting curve, we estimated the LoD of SARS-CoV-2 SCAN as 13.5 copies/ $\mu \mathrm{L}(22.5 \mathrm{aM})$ at a confidence level of $95 \%$.

\section{CONCLUSIONS}

In summary, we introduced and evaluated a sized counting method for nanopores-assisted CRISPR-Cas12a-based detection of SARS-CoV-2. A sized counting scheme for cleavage quantification of the reporters in the Cas12a assay was introduced by utilizing ECD values from the nanopore experiment. We found that the nanopore is a sensitive readout system to measure the cleavage ratios, a criterion used for positive or negative classification. A kinetic cleavage model was developed and experimentally validated to predict the reporter length distribution. This model revealed the trade-offs between sensitivity, reaction time, and false-positive rate in the SARSCoV-2 SCAN. These trade-offs could be relaxed by coupling with preamplification steps. With a 25 min RT-PCR step, 30 min of CRISPR Cas12a assay, and $10 \mathrm{~min}$ of nanopore reading (65 min of assay-to-result time), we achieved a limit of detection of 13.5 copies $/ \mu \mathrm{L}(22.5 \mathrm{aM})$ of viral RNA. The SARS-CoV-2 SCAN showed excellent specificity with no crossreactivity to other human coronaviruses. These results suggested that the solid-state CRISPR-Cas12a-assisted nanopores could provide a rapid, sensitive, and specific analysis of SARS-CoV-2.

\section{ASSOCIATED CONTENT}

\section{(s) Supporting Information}

The Supporting Information is available free of charge at https://pubs.acs.org/doi/10.1021/acs.nanolett.1c02974.

Methods section, including materials and chemicals, SARS-CoV-2 RT-PCR and Cas12a assay, glass nanopore fabrication and characterization, and nanopore sensing 
and data analysis sections. Nanopore characterization and current drop-dwell time measurements. Results of RT-qPCR. Gel electrophoresis results of the Cas12a assay products at five reaction times from 0.5 to $24 \mathrm{~h}$. Gel electrophoresis results of the no target negative control samples. Translocation recording of ssDNA reporters at different reaction times through the glass nanopore. Algorithm and the model details for estimation of the reporter length distribution over the trans-cleavage process. RT-PCR results for the analytical specificity and sensitivity test (PDF)

\section{AUTHOR INFORMATION}

\section{Corresponding Author}

Weihua Guan - Department of Electrical Engineering and Department of Biomedical Engineering, Pennsylvania State University, University Park, Pennsylvania 16802, United

States; (1) orcid.org/0000-0002-8435-9672;

Email: w.guan@psu.edu

\section{Authors}

Reza Nouri - Department of Electrical Engineering, Pennsylvania State University, University Park, Pennsylvania 16802, United States

Yuqian Jiang - Department of Biomedical Engineering and Huck Institutes of the Life Sciences, Pennsylvania State University, University Park, Pennsylvania 16802, United States

Zifan Tang - Department of Electrical Engineering, Pennsylvania State University, University Park, Pennsylvania 16802, United States

Xiaojun Lance Lian - Department of Biomedical Engineering, Huck Institutes of the Life Sciences, and Department of Biology, Pennsylvania State University, University Park, Pennsylvania 16802, United States

Complete contact information is available at: https://pubs.acs.org/10.1021/acs.nanolett.1c02974

\section{Author Contributions}

W.G. conceived the concept and together with X.L.L. supervised the study. R.N. and Z.T. designed and conducted the RT-PCR. R.N. and Y.J. developed and conducted the Cas12a assay. R.N. conducted the nanopore fabrication and sensing experiment. W.G. and R.N. cowrote the manuscript, with discussion from all authors.

\section{Notes}

The authors declare no competing financial interest.

\section{ACKNOWLEDGMENTS}

This work is supported by the National Science Foundation under Grant Nos. 1710831, 1912410, and 2045169. The content is solely the responsibility of the authors and does not necessarily represent the official views of the National Science Foundation. W.G. acknowledges the support from Coronavirus Research Seed Fund from PennState Huck Institutes of the Life Sciences \& Materials Research Institute.

\section{REFERENCES}

(1) Zhou, P.; Yang, X.-L.; Wang, X.-G.; Hu, B.; Zhang, L.; Zhang, W.; Si, H.-R.; Zhu, Y.; Li, B.; Huang, C.-L.; et al. A pneumonia outbreak associated with a new coronavirus of probable bat origin. Nature 2020, 579 (7798), 270-273.
(2) Zhu, N.; Zhang, D.; Wang, W.; Li, X.; Yang, B.; Song, J.; Zhao, X.; Huang, B.; Shi, W.; Lu, R.; et al. A novel coronavirus from patients with pneumonia in China, 2019. N. Engl. J. Med. 2020, 382, 727-733. (3) Lu, R.; Zhao, X.; Li, J.; Niu, P.; Yang, B.; Wu, H.; Wang, W.; Song, H.; Huang, B.; Zhu, N.; et al. Genomic characterisation and epidemiology of 2019 novel coronavirus: Implications for virus origins and receptor binding. lancet 2020, 395 (10224), 565-574.

(4) Chiara, M.; D’Erchia, A. M.; Gissi, C.; Manzari, C.; Parisi, A.; Resta, N.; Zambelli, F.; Picardi, E.; Pavesi, G.; Horner, D. S.; et al. Next generation sequencing of SARS-CoV-2 genomes: challenges, applications and opportunities. Briefings Bioinf. 2021, 22 (2), 616630.

(5) Aynaud, M.-M.; Hernandez, J. J.; Barutcu, S.; Braunschweig, U.; Chan, K.; Pearson, J. D.; Trcka, D.; Prosser, S. L.; Kim, J.; BarriosRodiles, M.; et al. A multiplexed, next generation sequencing platform for high-throughput detection of SARS-CoV-2. Nat. Commun. 2021, $12(1), 1405$.

(6) Bhoyar, R. C.; Jain, A.; Sehgal, P.; Divakar, M. K.; Sharma, D.; Imran, M.; Jolly, B.; Ranjan, G.; Rophina, M.; Sharma, S.; et al. High throughput detection and genetic epidemiology of SARS-CoV-2 using COVIDSeq next-generation sequencing. PloS one 2021, 16 (2), e0247115.

(7) Dzimianski, J. V.; Lorig-Roach, N.; O’Rourke, S. M.; Alexander, D. L.; Kimmey, J. M.; DuBois, R. M. Rapid and sensitive detection of SARS-CoV-2 antibodies by biolayer interferometry. Sci. Rep. 2020, 10 (1), 21738.

(8) Meng, Q.-B.; Peng, J.-J.; Wei, X.; Yang, J.-Y.; Li, P.-C.; Qu, Z.W.; Xiong, Y.-F.; Wu, G.-J.; Hu, Z.-M.; Yu, J.-C.; et al. Clinical application of combined detection of SARS-CoV-2-specific antibody and nucleic acid. World journal of clinical cases 2020, 8 (19), 4360.

(9) Petherick, A. Developing antibody tests for SARS-CoV-2. Lancet 2020, 395 (10230), 1101-1102.

(10) Udugama, B.; Kadhiresan, P.; Kozlowski, H. N.; Malekjahani, A.; Osborne, M.; Li, V. Y.; Chen, H.; Mubareka, S.; Gubbay, J. B.; Chan, W. C. Diagnosing COVID-19: the disease and tools for detection. ACS Nano 2020, 14 (4), 3822-3835.

(11) Jokela, P.; Jääskeläinen, A. E.; Jarva, H.; Holma, T.; Ahava, M. J.; Mannonen, L.; Lappalainen, M.; Kurkela, S.; Loginov, R. SARSCoV-2 sample-to-answer nucleic acid testing in a tertiary care emergency department: evaluation and utility. J. Clin. Virol. 2020, 131, 104614.

(12) Doudna, J. A.; Charpentier, E. The new frontier of genome engineering with CRISPR-Cas9. Science 2014, 346 (6213), 1258096.

(13) Gootenberg, J. S.; Abudayyeh, O. O.; Kellner, M. J.; Joung, J.; Collins, J. J.; Zhang, F. Multiplexed and portable nucleic acid detection platform with Cas13, Cas12a, and Csm6. Science 2018, 360 (6387), 439-444.

(14) Gootenberg, J. S.; Abudayyeh, O. O.; Lee, J. W.; Essletzbichler, P.; Dy, A. J.; Joung, J.; Verdine, V.; Donghia, N.; Daringer, N. M.; Freije, C. A.; et al. Nucleic acid detection with CRISPR-Cas13a/ C2c2. Science 2017, 356 (6336), 438-442.

(15) Abudayyeh, O. O.; Gootenberg, J. S.; Kellner, M. J.; Zhang, F. Nucleic acid detection of plant genes using CRISPR-Cas13. CRISPR Journal 2019, 2 (3), 165-171.

(16) Li, S.-Y.; Cheng, Q.-X.; Liu, J.-K.; Nie, X.-Q.; Zhao, G.-P.; Wang, J. CRISPR-Cas12a has both cis-and trans-cleavage activities on single-stranded DNA. Cell Res. 2018, 28 (4), 491-493.

(17) Bruch, R.; Baaske, J.; Chatelle, C.; Meirich, M.; Madlener, S.; Weber, W.; Dincer, C.; Urban, G. A. CRISPR/Cas13a-powered electrochemical microfluidic biosensor for nucleic acid amplificationfree miRNA diagnostics. Adv. Mater. 2019, 31 (51), 1905311.

(18) Shao, N.; Han, X.; Song, Y.; Zhang, P.; Qin, L. CRISPR-Cas12a Coupled with Platinum Nanoreporter for Visual Quantification of SNVs on a Volumetric Bar-Chart Chip. Anal. Chem. 2019, 91 (19), 12384-12391.

(19) Li, L.; Li, S.; Wu, N.; Wu, J.; Wang, G.; Zhao, G.; Wang, J. HOLMESv2: a CRISPR-Cas12b-assisted platform for nucleic acid detection and DNA methylation quantitation. ACS Synth. Biol. 2019, 8 (10), 2228-2237. 
(20) Qin, P.; Park, M.; Alfson, K. J.; Tamhankar, M.; Carrion, R.; Patterson, J. L.; Griffiths, A.; He, Q.; Yildiz, A.; Mathies, R.; et al. Rapid and fully microfluidic Ebola virus detection with CRISPRCas13a. ACS sensors 2019, 4 (4), 1048-1054.

(21) Kellner, M. J.; Koob, J. G.; Gootenberg, J. S.; Abudayyeh, O. O.; Zhang, F. SHERLOCK: nucleic acid detection with CRISPR nucleases. Nat. Protoc. 2019, 14 (10), 2986-3012.

(22) Yuan, C.; Tian, T.; Sun, J.; Hu, M.; Wang, X.; Xiong, E.; Cheng, M.; Bao, Y.; Lin, W.; Jiang, J.; et al. Universal and naked-eye gene detection platform based on the clustered regularly interspaced short palindromic repeats/cas12a/13a system. Analytical chemistry 2020, 92 (5), 4029-4037.

(23) Rauch, J. N.; Valois, E.; Solley, S. C.; Braig, F.; Lach, R. S.; Audouard, M.; Ponce-Rojas, J. C.; Costello, M. S.; Baxter, N. J.; Kosik, K. S.; et al. A scalable, easy-to-deploy protocol for Cas13-based detection of SARS-CoV-2 genetic material. J. Clin. Microbiol. 2021, 59 (4), e02402-20.

(24) Qian, C.; Wu, H.; Shi, Y.; Wu, J.; Chen, H. Dehydrated CRISPR-mediated DNA analysis for visualized animal-borne virus sensing in the unprocessed blood sample. Sens. Actuators, B 2020, 305, 127440 .

(25) Chenot, C. c.; Robiette, R. 1.; Collin, S. First evidence of the cysteine and glutathione conjugates of 3-sulfanylpentan-1-ol in hop (Humulus lupulus L.). J. Agric. Food Chem. 2019, 67 (14), 40024010.

(26) Sullivan, T. J.; Dhar, A. K.; Cruz-Flores, R.; Bodnar, A. G. Rapid, CRISPR-based, field-deployable detection of white spot syndrome virus in shrimp. Sci. Rep. 2019, 9 (1), 19702.

(27) Abudayyeh, O. O.; Gootenberg, J. S.; Konermann, S.; Joung, J.; Slaymaker, I. M.; Cox, D. B.; Shmakov, S.; Makarova, K. S.; Semenova, E.; Minakhin, L.; et al. C2c2 is a single-component programmable RNA-guided RNA-targeting CRISPR effector. Science 2016, 353 (6299), aaf5573.

(28) Patchsung, M.; Jantarug, K.; Pattama, A.; Aphicho, K.; Suraritdechachai, S.; Meesawat, P.; Sappakhaw, K.; Leelahakorn, N.; Ruenkam, T.; Wongsatit, T.; et al. Clinical validation of a Cas13-based assay for the detection of SARS-CoV-2 RNA. Nat. Biomed. Eng. 2020, 4 (12), 1140-1149.

(29) Harrington, L. B.; Burstein, D.; Chen, J. S.; Paez-Espino, D.; Ma, E.; Witte, I. P.; Cofsky, J. C.; Kyrpides, N. C.; Banfield, J. F.; Doudna, J. A. Programmed DNA destruction by miniature CRISPRCas14 enzymes. Science 2018, 362 (6416), 839-842.

(30) Nouri, R.; Tang, Z.; Dong, M.; Liu, T.; Kshirsagar, A.; Guan, W. CRISPR-based detection of SARS-CoV-2: A review from sample to result. Biosens. Bioelectron. 2021, 178, 113012.

(31) Broughton, J. P.; Deng, X.; Yu, G.; Fasching, C. L.; Servellita, V.; Singh, J.; Miao, X.; Streithorst, J. A.; Granados, A.; SotomayorGonzalez, A.; Zorn, K.; Gopez, A.; Hsu, E.; Gu, W.; Miller, S.; Pan, C.-Y.; Guevara, H.; Wadford, D. A.; Chen, J. S.; Chiu, C. Y. CRISPRCas12-based detection of SARS-CoV-2. Nat. Biotechnol. 2020, 38 (7), 870-874.

(32) Ali, Z.; Aman, R.; Mahas, A.; Rao, G. S.; Tehseen, M.; Marsic, T.; Salunke, R.; Subudhi, A. K.; Hala, S. M.; Hamdan, S. M.; et al. iSCAN: An RT-LAMP-coupled CRISPR-Cas12 module for rapid, sensitive detection of SARS-CoV-2. Virus Res. 2020, 288, 198129.

(33) Ding, X.; Yin, K.; Li, Z.; Lalla, R. V.; Ballesteros, E.; Sfeir, M. M.; Liu, C. Ultrasensitive and visual detection of SARS-CoV-2 using all-in-one dual CRISPR-Cas12a assay. Nat. Commun. 2020, 11 (1), 4711.

(34) Guo, L.; Sun, X.; Wang, X.; Liang, C.; Jiang, H.; Gao, Q.; Dai, M.; Qu, B.; Fang, S.; Mao, Y.; et al. SARS-CoV-2 detection with CRISPR diagnostics. Cell discovery 2020, 6 (1), 34.

(35) Arizti-Sanz, J.; Freije, C. A.; Stanton, A. C.; Petros, B. A.; Boehm, C. K.; Siddiqui, S.; Shaw, B. M.; Adams, G.; KosokoThoroddsen, T.-S. F.; Kemball, M. E.; et al. Streamlined inactivation, amplification, and Cas13-based detection of SARS-CoV-2. Nat. Commun. 2020, 11 (1), 5921.

(36) Abbott, T. R.; Dhamdhere, G.; Liu, Y.; Lin, X.; Goudy, L.; Zeng, L.; Chemparathy, A.; Chmura, S.; Heaton, N. S.; Debs, R.; et al.
Development of CRISPR as an antiviral strategy to combat SARSCoV-2 and influenza. Cell 2020, 181 (4), 865-876.

(37) Wang, R.; Qian, C.; Pang, Y.; Li, M.; Yang, Y.; Ma, H.; Zhao, M.; Qian, F.; Yu, H.; Liu, Z.; et al. opvCRISPR: One-pot visual RTLAMP-CRISPR platform for SARS-cov-2 detection. Biosens. Bioelectron. 2021, 172, 112766

(38) Ning, B.; Yu, T.; Zhang, S.; Huang, Z.; Tian, D.; Lin, Z.; Niu, A.; Golden, N.; Hensley, K.; Threeton, B.; et al. A smartphone-read ultrasensitive and quantitative saliva test for COVID-19. Science advances 2021, 7 (2), eabe 3703.

(39) Chen, J. S.; Ma, E.; Harrington, L. B.; Da Costa, M.; Tian, X.; Palefsky, J. M.; Doudna, J. A. CRISPR-Cas12a target binding unleashes indiscriminate single-stranded DNase activity. Science 2018, 360 (6387), 436-439.

(40) Fologea, D.; Brandin, E.; Uplinger, J.; Branton, D.; Li, J. DNA conformation and base number simultaneously determined in a nanopore. Electrophoresis 2007, 28 (18), 3186-3192.

(41) Fologea, D.; Gershow, M.; Ledden, B.; McNabb, D. S.; Golovchenko, J. A.; Li, J. Detecting single stranded DNA with a solid state nanopore. Nano Lett. 2005, 5 (10), 1905-1909.

(42) Grosberg, A. Y.; Rabin, Y. DNA capture into a nanopore: interplay of diffusion and electrohydrodynamics. J. Chem. Phys. 2010, 133 (16), 165102.

(43) Albrecht, T. Single-molecule analysis with solid-state nanopores. Annu. Rev. Anal. Chem. 2019, 12, 371-387.

(44) Wanunu, M.; Sutin, J.; McNally, B.; Chow, A.; Meller, A. DNA translocation governed by interactions with solid-state nanopores. Biophys. J. 2008, 95 (10), 4716-4725.

(45) Nouri, R.; Tang, Z.; Guan, W. Calibration-free nanopore digital counting of single molecules. Anal. Chem. 2019, 91 (17), 1117811184

(46) Lu, X.; Wang, L.; Sakthivel, S. K.; Whitaker, B.; Murray, J.; Kamili, S.; Lynch, B.; Malapati, L.; Burke, S. A.; Harcourt, J.; et al. US CDC real-time reverse transcription PCR panel for detection of severe acute respiratory syndrome coronavirus 2 . Emerging Infect. Dis. 2020, 26 (8), 1654.

(47) Duke, T.; Viovy, J. L.; Semenov, A. N. Electrophoretic mobility of DNA in gels. I. New biased reptation theory including fluctuations. Biopolymers 1994, 34 (2), 239-247.

(48) Nouri, R.; Jiang, Y.; Lian, X. L.; Guan, W. Sequence-specific recognition of HIV-1 DNA with solid-state CRISPR-Cas12a-assisted nanopores (SCAN). ACS sensors 2020, 5 (5), 1273-1280.

(49) Ramachandran, A.; Santiago, J. G. CRISPR Enzyme Kinetics for Molecular Diagnostics. Anal. Chem. 2021, 93 (20), 7456-7464.

(50) Alonso Villela, S. M.; Kraiem, H.; Bouhaouala-Zahar, B.; Bideaux, C.; Aceves Lara, C. A.; Fillaudeau, L. A protocol for recombinant protein quantification by densitometry. MicrobiologyOpen 2020, 9 (6), 1175-1182.

(51) McKenzie, B.; Kay, G.; Matthews, K. H.; Knott, R. M.; Cairns, D. The hen's egg chorioallantoic membrane (HET-CAM) test to predict the ophthalmic irritation potential of a cysteamine-containing gel: Quantification using Photoshop ${ }^{\circledR}$ and ImageJ. Int. J. Pharm. 2015, 490 (1-2), 1-8.

(52) Benschop, K.; Molenkamp, R.; van der Ham, A.; Wolthers, K.; Beld, M. Rapid detection of human parechoviruses in clinical samples by real-time PCR. J. Clin. Virol. 2008, 41 (2), 69-74.

(53) Holstein, C. A.; Griffin, M.; Hong, J.; Sampson, P. D. Statistical method for determining and comparing limits of detection of bioassays. Anal. Chem. 2015, 87 (19), 9795-9801.

(54) Forootan, A.; Sjöback, R.; Björkman, J.; Sjögreen, B.; Linz, L.; Kubista, M. Methods to determine limit of detection and limit of quantification in quantitative real-time PCR (qPCR). Biomolecular detection and quantification 2017, 12, 1-6. 